\title{
Flow in re-stenosed artery after angioplasty
}

\author{
S. I. Bernad ${ }^{1}$, A. Totorean ${ }^{2}$, E. S. Bernad ${ }^{3}$ \& R. Susan-Resiga ${ }^{2}$ \\ ${ }^{1}$ Romanian Academy, Timisoara Branch, Romania \\ 2 "Politehnica" University of Timisoara, Romania \\ ${ }^{3}$ University of Medicine and Pharmacy Timisoara, Romania
}

\begin{abstract}
Coronary angiogram (CA) is the gold standard diagnostic tool for coronary artery stenosis. Restenosis after balloon angioplasty is the major limitation of the technique. The goal of the paper is to evaluate the way in which the wall shear stress (WSS) distribution is affected by the blood flow evolution during the cardiac cycle in re-stenosed arterial segment. Coronary angiography was performed 2 months after percutaneous transluminal coronary angioplasty (PTCA). Angiographic evidence of restenosis was defined as $40 \%$ diameter stenosis at the site of the previous dilatation. After angioplasty of stenotic lesions, the luminal geometry is drastically changed. The maximum value of the WSS is at the critical height of the stenosis. A large separation zone is observed downstream to the stenosis, characterized by low WSS region. The range of WSS varies from 2 to $24 \mathrm{~Pa}$. In conclusion, the arterial remodeling is highly relevant to the process of re-stenosis and is an important adaptive response to changes in flow and local shear stress.

Keywords: blood flow, right coronary artery, angioplasty, hemodynamics, pressure drop.
\end{abstract}

\section{Introduction}

It is widely believed that atherosclerosis development and progression are affected by many risk factors, such as static pressure, wall shear stress, blood viscosity, flow velocity, etc. Vascular geometry is another factor influencing strongly the flow patterns and generating a pre-existing atherogenic hemodynamic environment [1, 2].

When atherosclerosis develops, advanced lesions form first in some regions with adaptive intimal thickening. 
Adaptive intimal thickening may be thought of as the consequence of a range of physiological stimuli, constituting an attempt by the tissue to maintain normal conditions of flow, wall tension, or both. Intima, as a living, reactive tissue, adapts in thickness to changes in pulse rate, blood pressure, arterial geometry, flow rate, and resistance to flow in distal vascular segments and in supplied organs [3, 4].

The flow phenomena in the stenotic arteries include asymmetric flow, instabilities and laminar to turbulent transition [5]. The unsteady flow in a stenotic artery also is characterized by high pressure and wall shear stress (WSS) in the throat and distal to the stenosis [6].

Coronary angiogram is the gold standard diagnostic tool for coronary artery stenosis.

Restenosis after balloon angioplasty (BA) is the major limitation of the technique. Excessive neointimal proliferation and extracellular matrix synthesis by modified smooth muscle cells in response to injury have been suggested as the main mechanisms of restenosis [7].

The goal here is twofold. First the velocity and pressure distributions at the locations of constricted region are evaluated. Second the way in which the WSS distribution is affected by the blood flow evolution during the cardiac cycle in stenosed region are investigated.

\section{Materials and method}

\subsection{Quantitative angiography}

In total, three coronary angiograms are obtained for the patient: before and after percutaneous transluminal coronary angioplasty (PTCA) and at angiographic follow-up.

The procedure was considered successful when the residual luminal narrowing in the dilated segment immediately after angioplasty was $<50 \%$ and when no major complication (electrocardiography - ECG or enzymatic evidence of myocardial infarction) occurred.

A suboptimal result was defined as the presence of a visually estimated residual stenosis $>40 \%$ after dilatation with or without an associated nonocclusive dissection but with normal blood flow. The anatomical and geometric measurement is performed by contrast angiography.

\subsubsection{Patient follow-up}

Coronary angiography techniques are performed 2 months after PTCA in order to provide hemodynamic and anatomic data of the right coronary artery (RCA). Angiographic evidence of restenosis was defined as $40 \%$ diameter stenosis at the site of the previous dilatation. The degree of stenosis was evaluated by quantitative coronary angiography with automatic edge detection. Calibration of the diameter of the vessels in absolute values (in millimeters) was achieved with the diagnostic or guiding catheter used as a reference. Measurements were repeated three times, and the mean values were recorded. 


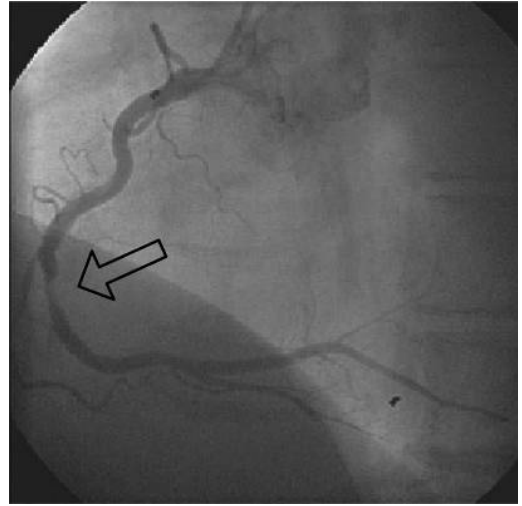

(a)

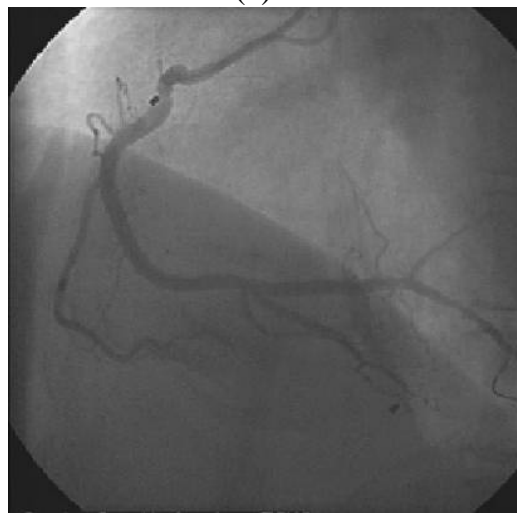

(c)

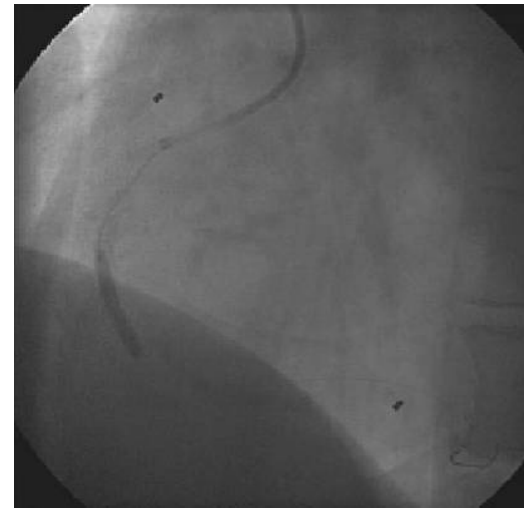

(b)

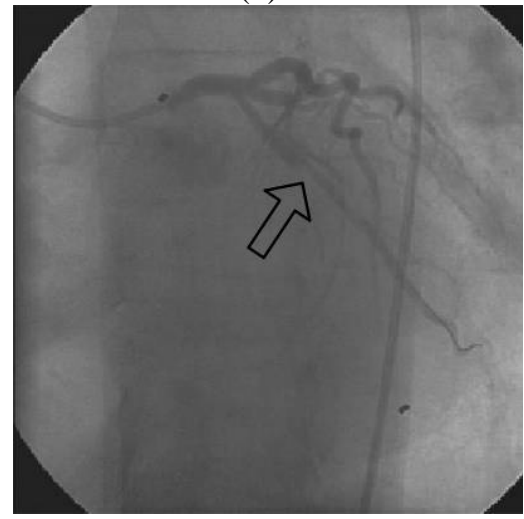

(d)

Figure 1: Invasive coronary angiography and angioplasty on the right coronary artery (RCA). (a) RCA mid segment stenosis (angiographic image); (b) balloon inflation during the angioplasty; (c) RCA after angioplasty; (d) RCA restenosis at 2 months after intervention (angiographic image).

Table 1: $\quad$ Baseline clinical characteristics of the patient before CA.

\begin{tabular}{lc}
\hline \multicolumn{1}{c}{ Patient data } & Characteristics \\
\hline Sex & M \\
Age, (y) & 52 \\
Smoker & Yes \\
Previous myocardial infarction & No \\
Previous CABG & No \\
Previous PTCA & No \\
Diabetes mellitus & No \\
History of hypertension & Yes \\
History of hypercholesterolemia & Yes \\
History of peripheral vascular disease & No \\
\hline
\end{tabular}


Clinical characteristics of patient before coronary angiography are presented in Table 1, and angiographic data obtained during the coronary intervention are presented in Table 2.

Table 2: Baseline angiographic data of patient before coronary angioplasty.

\begin{tabular}{|c|c|}
\hline Angiographic data & Characteristics \\
\hline \multicolumn{2}{|l|}{ Number of diseased vessels } \\
\hline SVD & Yes \\
\hline $2-\mathrm{VD}$ & - \\
\hline $3-V D$ & - \\
\hline \multicolumn{2}{|l|}{ Lesion location } \\
\hline LAD & - \\
\hline LCx & - \\
\hline RCA & Yes \\
\hline \multicolumn{2}{|l|}{ Type of lesion } \\
\hline Multiple irregularities & Yes \\
\hline Side branch in lesion & - \\
\hline Lesion calcification & - \\
\hline \multicolumn{2}{|l|}{ PTCA procedure } \\
\hline Nominal size of largest balloon [mm] & - \\
\hline Balloon to artery ratio & Yes \\
\hline Total number of inflations & 1 \\
\hline Total duration of inflation [s] & 84 \\
\hline Maximum inflation pressure [atm] & 6 \\
\hline \multicolumn{2}{|l|}{ Post-PTCA result } \\
\hline Dissection at the dilated site & No \\
\hline Thrombus visible (before or after PTCA) & No \\
\hline \multicolumn{2}{|l|}{ Reference diameter [mm] } \\
\hline Before & 3.01 \\
\hline After & 3.02 \\
\hline \multicolumn{2}{|l|}{ Minimal luminal diameter [mm] } \\
\hline Before & 1.2 \\
\hline After & 2.7 \\
\hline \multicolumn{2}{|l|}{ Diameter stenosis [\%] } \\
\hline Before & 60 \\
\hline After & 10 \\
\hline
\end{tabular}

\subsection{Vascular model}

Figure 2 shows the reconstructed lumen geometry of the RCA. Anatomical measurements are performed by contrast angiography. The lumen inlet diameter, $\left(d_{e}\right)$ in equal to $3 \mathrm{~mm}$ and the diameter of the lumen in the "throat" of the stenosis, $\left(\mathrm{d}_{\mathrm{t}}\right)$ is equal to $1.8 \mathrm{~mm}$.

SVD indicates single-vessel disease; 2-VD, two-vessel disease; 3-VD, threevessel disease; LAD - left anterior descending; LCx - left circumflex artery; RCA - right coronary artery; and PTCA - percutaneous transluminal coronary angioplasty, CABG - coronary artery bypass graft. 
The percentage diameter reduction for a circular stenosis is $1-d_{t} / d_{e}$ and the diameter of the occluded artery $\left(d_{r}\right)$ is equal to $\left(d_{e}\right)$. The RCA is modelled to be $20 \mathrm{~mm}$ in length with variable diameters, depending of the stenosis severity. Data corresponding to the reconstructed stenosis is presented in Figure 2.

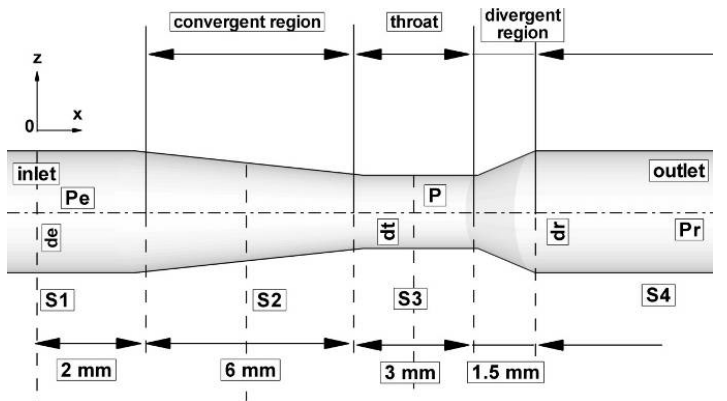

Figure 2: Dimensions of the stenosis at follow-up investigation. Patient specific RCA geometry reconstruction (corresponding to the stenosed region). S1-S4, correspond to the different investigated sections. Geometric measurements are performed by contrast angiography.

\subsection{Numerical model}

Three different meshes were investigated. The number of cells varied between 200,000 and 700,000. A high mesh resolution near the walls was needed for accurate values of pressure drop and WSS (Figure 3(a)). Thus the mesh was refined in the near-wall region. To model the flow close to the wall, standard wall-function approach was used.

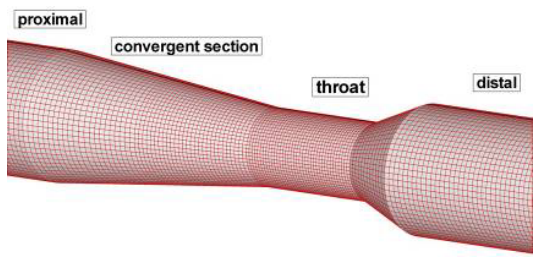

(a)

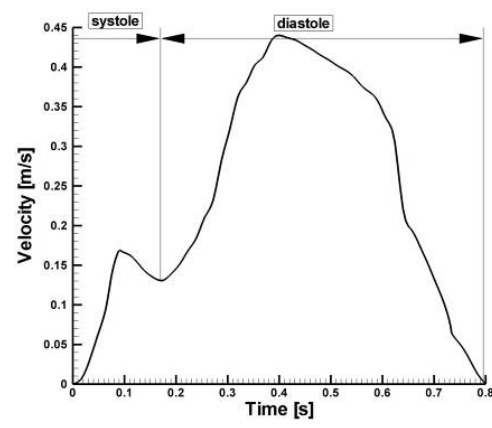

(b)

Figure 3: (a) Computational domain discretization; (b) Physiologically realistic boundary conditions based on flow waveform acquired with an intravascular ultrasound Doppler probe in the RCA. diastolic dominant flow waveform. 
The governing equations are solved iteratively until convergence of all flow variables is achieved. The convergence criterion was set to $10^{-6}$ for the residuals of the continuity equation and of $X, Y$ and $Z$ momentum equations.

Computational assumptions and boundary conditions are the following: the blood is assumed incompressible having dynamic viscosity $(\mu)$ of 0.00408 Pa.s and a density $(\rho)$ of $1050 \mathrm{~kg} / \mathrm{m}^{3}$.

Calculated Womersley numbers Wo $=\mathrm{R}(2 \pi \mathrm{f} \rho / \mu)^{0.5}$ (where $R=3 \mathrm{~mm}$ is the artery radius and $f=72 \mathrm{bpm}$ is the heart frequency) were found to be low in coronary arteries (Wo $=4.17)$. The blood flow velocity profile in the human artery tends to be laminar and parabolic because of the friction (wall shear stress) between the viscous blood and the inner surface of blood vessel [8].

The governing equations for incompressible blood flow are the Navier-Stokes equations and are solved iteratively until obtain the required numerical precision. The numerical simulations are performed using the commercial CFD package FLUENT 6.3 [9]. The velocity profile at the inlet section is identical to the real coronary ostium velocity profile presented in Figure 3(b).

In this model the vessel wall is a rigid wall. Researchers from different groups $[10,11]$ conclude that arterial wall motion had little effect on the hemodynamic parameters distributions within the RCA.

\section{Results}

During clinical angioplasty, balloon sizes, inflation pressures, and time could also influence the extent and shape of vessel wall injury. However, assuming the balloon is appropriately sized and inflation pressure is adequately applied, balloon inflation exerts a relatively uniform tensile stress along the entire circumference of the lesion. The physical structure and property of the lesion determine where the stretching or splitting will occur [12].

The numerical simulation result show the hemodynamic parameters of the blood flow in the re-stenosed coronary artery under physiological conditions (velocity, pressure and wall shear stress distribution).

\subsection{Velocity distribution}

Variation of axial velocity profiles during the cardiac cycle at progressive locations along the lesion are in Figure 4 at mean flow rates $t=0.4 \mathrm{~s}$ in Figure 3(b).

Along the constriction region where spatial flow acceleration occurs, the velocity profiles at the midpoint are steeper in the wall region and somewhat more uniform in the higher velocity core flow, although the shapes of the profiles vary during the cardiac cycle, and with mean flow rate.

In the throat region where mean flow velocities are highest along the lesion, the velocity profiles at the midpoint indicate that viscous effects are more confined to the near wall region with a more uniform core flow (like boundary layer type flow) during the cardiac cycle (Figure 5). 


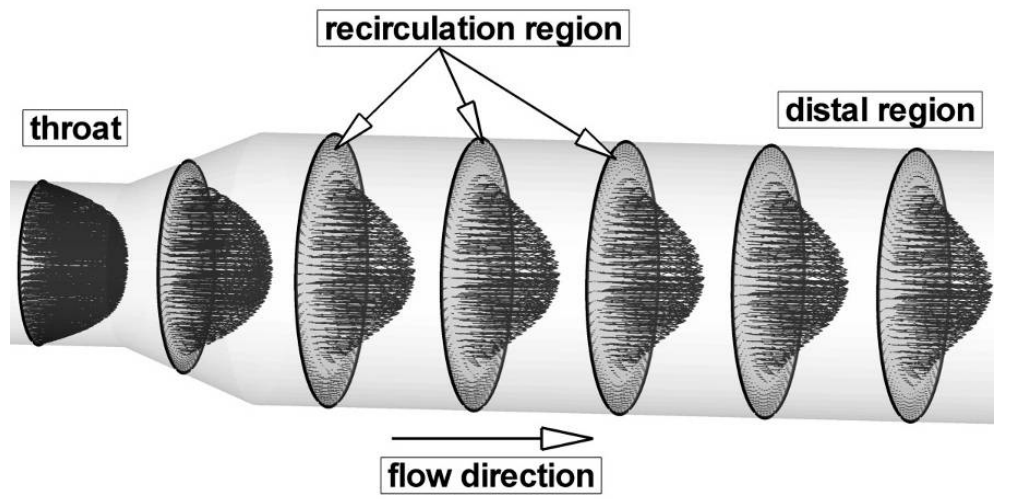

Figure 4: Velocity vector distribution in different cross sections in distal part of the re-stenosed RCA artery.

\subsection{Pressure distribution}

First, the flow enter in the constriction and the increase in velocity (Figure 5) results in a steep drop in the pressure coefficient which reaches the minimum value at the constricted region (Figure 6). Beyond this point, the flow enters the expansion and the pressure coefficient exhibits a steep increase.

Separation of flow can also contribute to pressure loss and is a major factor at lower percent stenoses. A strong shear layer at the interface of the separation region and the central jet creates additional viscous losses. The throat of the stenosis is a site of high shear stress rates at the wall, which also contribute to viscous losses.

\subsection{Wall shear stress distribution}

A primary signal for arterial remodeling is shear stress, which is the frictional force at the endothelial surface produced by flowing blood [13, 14].

Because the vessel narrows at the stenosis, the velocity at the center of the vessel increases due to the conservation of mass law. The velocity gradient at the wall and the resulting WSS is maximum in the narrowest portion of the stenosis. Figure 7(b) shows the calculated WSS distribution variation along the stenosis.

The artery radius starts at $3 \mathrm{~mm}$, gradually narrows to $1.8 \mathrm{~mm}$ at the stenosis, and then expands after the stenosis to about $3 \mathrm{~mm}$. Along to the arterial wall, the WSS varies significantly from point to point. The WSS is low outside the stenosis, suddenly increases with the narrowing diameter, and reaches a maximum at the smallest diameter inside the stenosis (Figure 7(b)). Distal to the sudden expansion of the stenosis, the vortex backflow produces low WSS values.

The maximum value of wall shear stress is at the critical height of the stenosis (Figure 7(b)). A large separation zone is observed also downstream to the stenosis (characterized by low WSS region). 

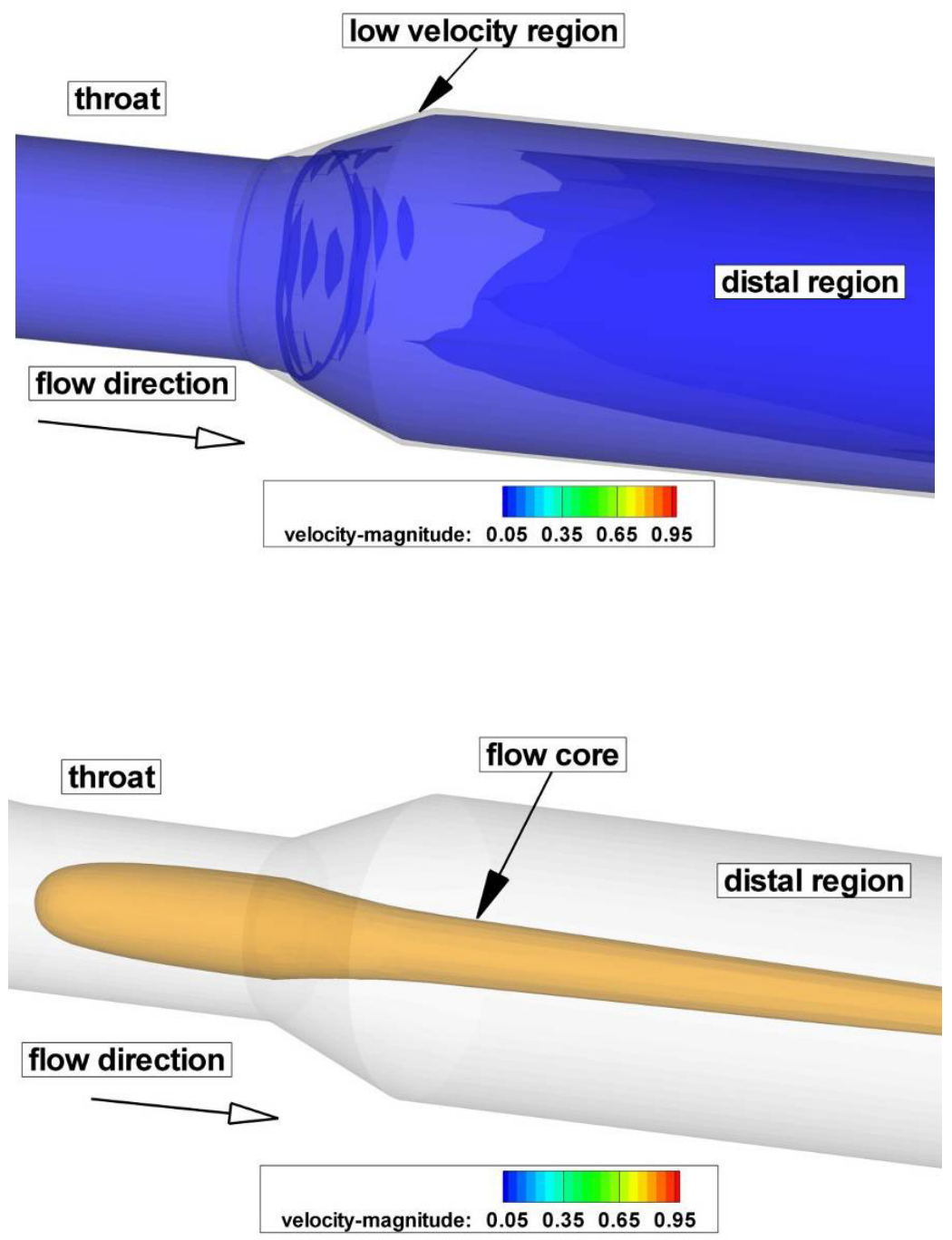

Figure 5: Iso-velocity distribution for time step $\mathrm{t}=0.64 \mathrm{~s}$ (diastolic decceleration phase) in the investigated stenosis (time step correspond to the time steps indicated in Figure 3(b)). Top: isovelocity contour for velocity of $0.02 \mathrm{~m} / \mathrm{s}$; bottom: iso-velocity contour for velocity of $0.9 \mathrm{~m} / \mathrm{s}$.

The range of WSS in this figure varies from 2 to $24 \mathrm{~Pa}$. Large intense regions of low WSS appear downstream at the stenosis. For this region the WSS is approximately $2 \mathrm{~Pa}$. The maximum WSS in the stenosis is about $24 \mathrm{~Pa}$. 


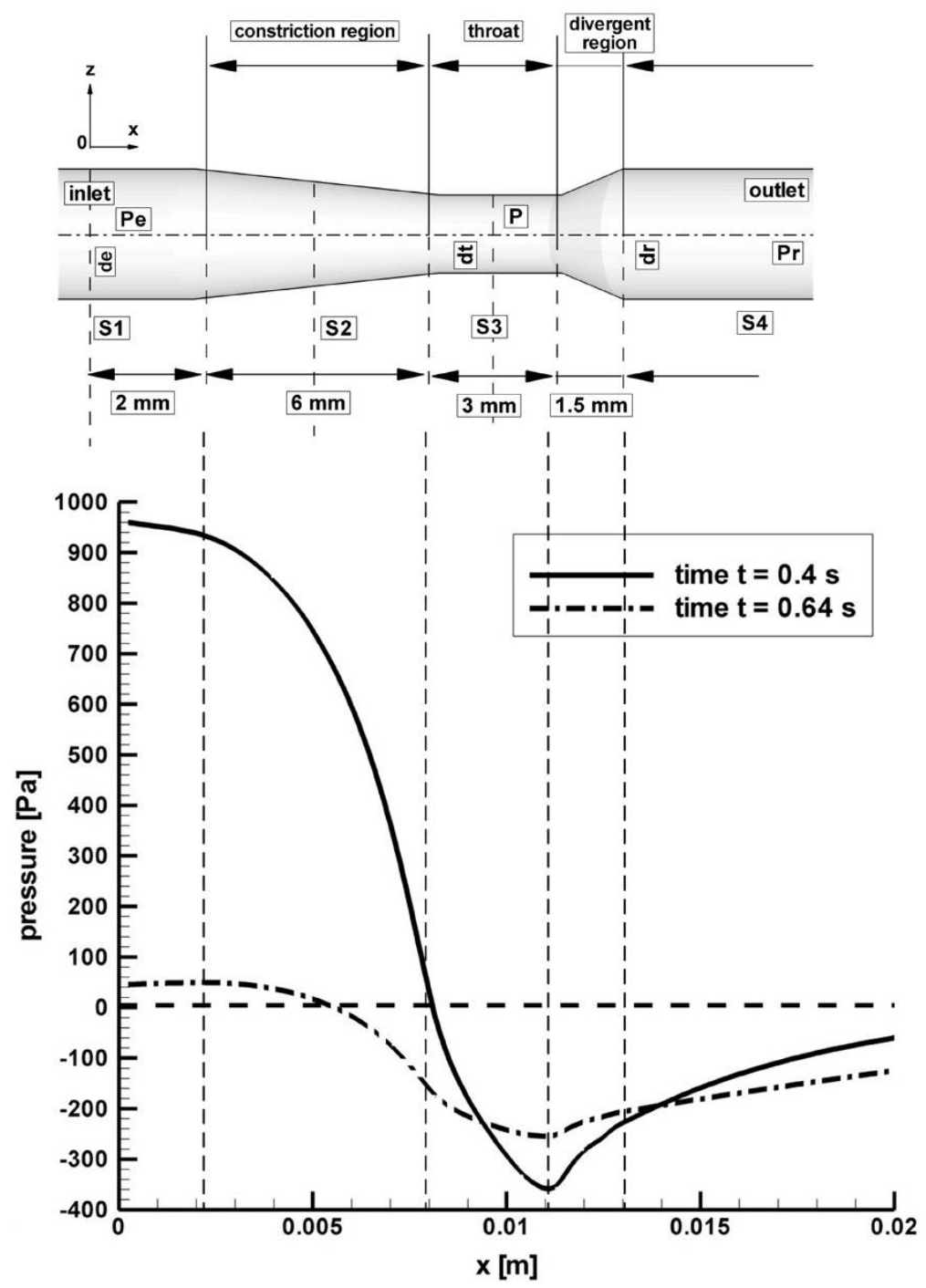

Figure 6: Axial pressure distribution for different time steps in re-stenosed RCA.

\section{Conclusion}

Arterial remodeling is highly relevant to the process of atherosclerosis and is an important adaptive response to changes in flow and local shear stress.

Restenosis is the result of the interaction between different biologic determinants. We conclude that one major determinant that influence the 


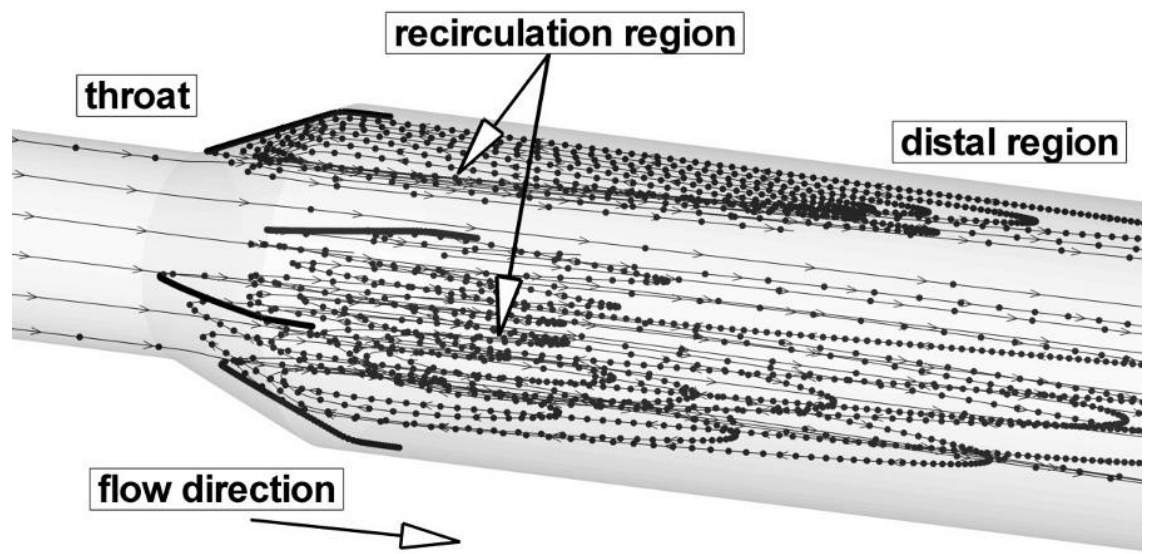

(a)

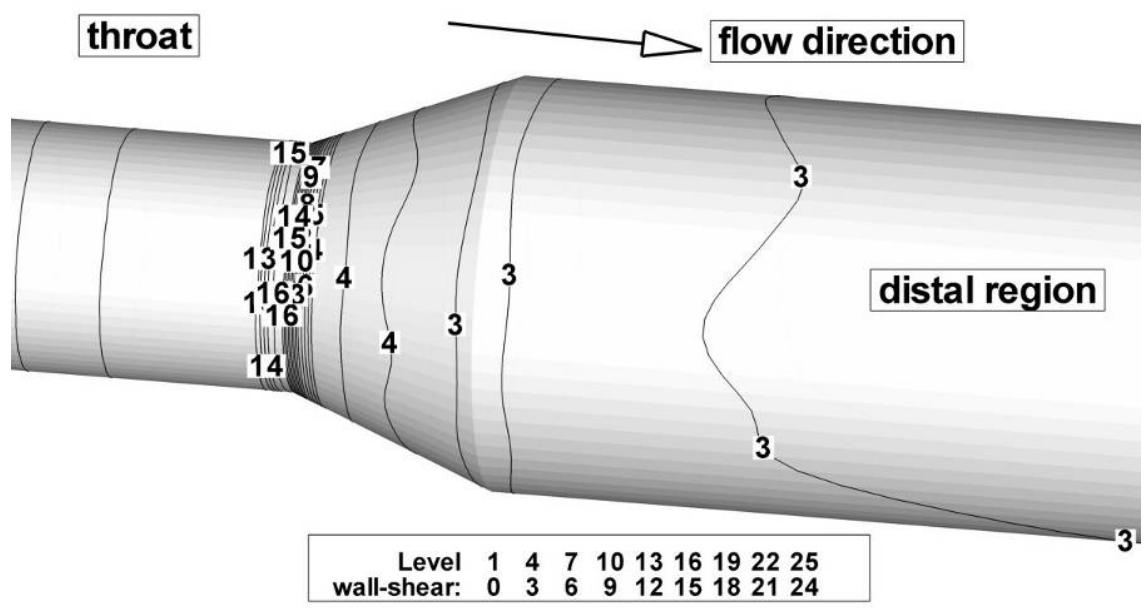

(b)

Figure 7. Disturbed flow indicators at the time $t=0.64 \mathrm{~s}$. (a) particles pattline shows large recirculation region distal to the stenotic segment; (b) temporal WSS magnitude contours around to the throat (value are in [Pa]).

restenosis is the regional flow characteristics (determined by the geometry of the dilated lumen of the lesion and blood flow velocity patterns across that lumen).

In the clinical setting, a less than satisfactory residual lumen after coronary angioplasty or a postdilated residual stenosis greater than $30 \%$ is associated with increased restenosis [15]. This is probably because a larger postdilation residual stenosis tends to be associated with a greater flow separation area (were the low shear stress promote intimal growth). 
With regard to fluid dynamics in the case of poststenotic regions, the areas of low wall shear stress are also associated with flow separation. This is important because it has been suggested that the fluctuation of wall shear stress or existence of the disturbed flow leads to increased endothelial cell turnover and intimal thickening $[16,17]$.

\section{Glossary}

BA - balloon angioplasty,

CA - coronary angiogram,

CABG - coronary artery bypass graft,

ECG - electrocardiography,

LAD - left anterior descending,

LCx - left circumflex artery,

RCA - right coronary artery,

PTCA - percutaneous transluminal coronary angioplasty,

SVD - single-vessel disease,

2-VD - two-vessel disease,

3-VD - three-vessel disease,

WSS - wall shear stress.

\section{References}

[1] S.R. Dodds, The haemodynamics of asymmetric stenoses, European Journal of Vascular and Endovascular Surgery, 24, 332-337, 2002.

[2] D. Zeng, E. Boutsianis, M. Ammann, K. Boomsma, S. Wildermuth, D. Poulikakos, A study of the compliance of a right coronary artery and its impact on wall shear stress, Journal of Biomechanical Engineering, 130, 041014-11, 2008.

[3] P. H. Stone, A. U. Coskun, S. Kinlay, et al, Regions of low endothelial shear stress are the sites where coronary plaque progresses and vascular remodelling occurs in humans: an in vivo serial study, Eur Heart J, 28, 705-710, 2007.

[4] H.C. Stary, D.H. Blankenhorn, A.B. Chandler, S. Glagov, W. Insull, Jr. M. Richardson, A definition of the intima of human arteries and of its atherosclerosis- prone regions. A report from the Committee on Vascular Lesions of the Council on Arteriosclerosis, American Heart Association, Circulation, 85, 391-405, 1992.

[5] J.R Buchanan., C. Kleinstreuer, Simulation of particle-hemodynamics in a partially occluded artery segment with implications to the initiation of microemboli and secondary stenoses, Journal of Biomechanical Engineering, 120, 446-454, 1998.

[6] J.-J. Ciu, D.L. Wang, S. Chien, R. Skalak, S. Usami, Effects of disturbed flow on endothelial cells, Journal of Biomechanical Engineering, 120, 2-8, 1988. 
[7] G.E. Austin, N.B. Ratliff, J. Hollman, S. Tabei, D.F. Philips, Intimal proliferation of smooth muscle cells as an explanation for recurrent coronary artery stenosis after percutaneous transluminal coronary angioplasty, J Am Coll Cardiol, 6, 369-375, 1985.

[8] A.M. Malek, S.L. Alper, S. Izumo, Hemodynamic shear stress and its role in atherosclerosis, Jama, 282, 2035-42, 1999.

[9] FLUENT 6.3 User's Guide, Ansys Fluent Incorporated, 2006.

[10] Y. Qiu, J.M. Tarbell, Numerical simulation of pulsatile flow in a compliant curved tube model of a coronary artery. J. Biomech. Eng., 122, 77-85, 2000.

[11] A. Santamarina, E. Weydahl, J.M. Siegel Jr., J.E. Moore Jr., Computational analysis of flow in a curved tube model of the coronary arteries: Effects of time-varying curvature, Ann. Biomed. Eng., 26, 944-954, 1998.

[12] R.T. Lyon, C.K. Zarins, C.-T. Lu, C.-F. Yang, S. Glagov, Vessel, plaque and lumen morphology after transluminal balloon angioplasty: Quantitative study in distended human arteries, Arteriosclerosis, Thrombosis and Vascular Biology, 7, 306-314, 1987.

[13] M. Heil, W. Schaper, Influence of mechanical, cellular, and molecular factors on collateral artery growth (arteriogenesis). Circ Res., 95, 449-458, 2004.

[14] N. Resnick, H. Yahav, A. Shay-Salit, M. Shushy, S. Schubert, L.C. Zilberman, E. Wofovitz, Fluid shear stress and the vascular endothelium: for better and for worse. Prog Biophys Mol Biol., 811, 177-199, 2003

[15] P.P. Leimgruber, G.S. Roubin, J. Hollman, G.A. Cotsonis, B. Meier, J.S. Jr Douglas, S.B. III King, A.R. Gruentzig, Restenosis after successful coronary angioplasty in patients with single vessel disease, Circulation, 73, 710-717, 1986.

[16] C.K. Zarins, R.A. Bomberger, S. Glagov, Local effects of stenoses: Increased flow velocity inhibits atherogenesis, Circulation, 64 (suppl II): II-221-II-227, 1981.

[17] B.L. Langille, F. O’Donnell, Reductions in arterial diameter produced by chronic decrease in blood flow are endotheliumdependent, Science, 231, 405-407, 1986. 